\title{
TIMELIKE ROTATIONAL SURFACES WITH LIGHTLIKE PROFILE CURVE
}

\author{
ERHAN GÜLER AND H. HILMI HACISALIHOĞLU
}

\begin{abstract}
In this work, some geometric properties of the timelike rotational surfaces with lightlike profile curve of $(S, L),(T, L)$ and $(L, L)$ - types are shown in Minkowski 3-space.
\end{abstract}

\section{Introduction}

Rotational surfaces in Euclidean 3-space have been studied for a long time and many examples of such surfaces have been discovered. On the other hand, Minkowski 3-space has more complicated geometric structures compared to Euclidean 3-space. In particular, Minkowski 3-space has distinguished axes of rotation, namely, spacelike, timelike and lightlike (or null) axes. About the semi (proper) Riemannian geometry, many nice books have been done such as [4], [5] and [9].

If we focus on the ruled (helicoid) and rotational characters, we have Bour's theorem in [2].

Ikawa determined pairs of surfaces by Bour's theorem with the additional condition that they have the same Gauss map in Euclidean 3-space in [6]. Ikawa classified the spacelike and timelike surfaces as (axis, profile curve)-type in [7]. He proved an isometric relation between a spacelike (timelike) generalized helicoid and a spacelike (timelike) rotational surface of spacelike (timelike) axis of $(S, S),(S, T),(T, S)$ and $(T, T)$ - types by Bour's theorem in Minkowski 3-space.

Güler [3] showed that a generalized helicoid and a rotational surface with lightlike profile curve have an isometric relation by Bour's theorem in Minkowski 3-space. He classified the spacelike (and timelike) helicoidal (and rotational) surfaces with lightlike profile curve of $(S, L),(T, L)$ and $(L, L)$-types.

Consider a smooth surface $\mathbf{M}$ in $\mathbb{R}_{1}^{3}$ is described locally by an isometric immersion

$$
R: \mathbf{D} \subset \mathbb{R}^{2} \longrightarrow \mathbb{R}_{1}^{3}
$$

Received by the editors Dec. 12, 2010, Accepted:May 30, 2011.

2000 Mathematics Subject Classification. Primary 53A35; Secondary 53C45.

Key words and phrases. Rotational surface, null profile curve, Gauss map, mean curvature, Gaussian curvature. 
where $(u, v)$ are local coordintes on the simply connected and bounded domain $\mathbf{D}$. Let $\mathbf{N}=\mathbf{N}(u, v)$ be the unit normal vector field on $\mathbf{M}$.

In local coordinates $\left(x_{1}, x_{2}\right)$ for a surface $\mathbf{M}$ furnished with pseudo-Riemannian metric say $g$ given by its matrix $\left(g_{i j}\right)$ with inverse matrix $\left(g^{i j}\right)$, the Laplacian $\Delta$ of $\mathbf{M}$ is

$$
\Delta=-\frac{1}{\sqrt{\left|\operatorname{det}\left(g_{i j}\right)\right|}} \sum \frac{\partial}{\partial x_{i}}\left(\sqrt{\left|\operatorname{det}\left(g_{i j}\right)\right|} g^{i j} \frac{\partial}{\partial x_{j}}\right)
$$

in [8]. Moreover, the Laplace-Beltrami operator of a function $\phi=\left.\phi(u, v)\right|_{\mathbf{D}}$ with respect to the first fundamental form of $\mathbf{M}$ is defined by [1] as follow

$$
\Delta \phi=-\frac{1}{\sqrt{E G-F^{2}}}\left[\left(\frac{G \phi_{u}-F \phi_{v}}{\sqrt{E G-F^{2}}}\right)_{u}-\left(\frac{F \phi_{u}-E \phi_{v}}{\sqrt{E G-F^{2}}}\right)_{v}\right]
$$

where $(u, v)$ are local coordintes on $\mathbf{D}$.

Let $\mathbf{F}(u, v)=\left(f_{1}(u, v), f_{2}(u, v), f_{3}(u, v)\right)$ be a vector function defined on the domain $\mathbf{D}$, then we set

$$
\Delta \mathbf{F}(u, v)=\left(\Delta f_{1}(u, v), \Delta f_{2}(u, v), \Delta f_{3}(u, v)\right) .
$$

In this paper, we give some geometric relations of the timelike rotational surfaces $(T R S)$ with lightlike profile curve $(L P C)$ of $(S, L),(T, L)$ and $(L, L)$-types in Minkowski 3-space. In section 2, we recall some basic notions of the Lorentzian geometry and give the definition of the rotational surfaces. TRS with LPC are given to find some geometric properties in section 3. In section 4, we study some relations between Gauss maps and Laplace-Beltrami operator of the Gauss maps, the curvatures of the TRS with LPC.

\section{Preliminaries}

In this section, we will obtain some rotational surfaces with lightlike profile curve in Minkowski 3 -space. In the rest of this paper we shall identify a vector $(a, b, c)$ with its transpose $(a, b, c)^{t}$.

The Minkowski 3 -space $\mathbb{R}_{1}^{3}$ is the Euclidean space $\mathbb{E}^{3}$ provided with the inner product

$$
\langle\vec{p}, \vec{q}\rangle_{L}=p_{1} q_{1}+p_{2} q_{2}-p_{3} q_{3}
$$

where $\vec{p}=\left(p_{1}, p_{2}, p_{3}\right), \vec{q}=\left(q_{1}, q_{2}, q_{3}\right) \in \mathbb{R}^{3}$. We say that a Lorentzian vector $\vec{p}$ in $\mathbb{R}_{1}^{3}$ is spacelike (resp. lightlike and timelike) if $\vec{p}=0$ or $\langle\vec{p}, \vec{p}\rangle_{L}>0$ (resp. $\vec{p} \neq 0 ;\langle\vec{p}, \vec{p}\rangle_{L}=0$ and $\left.\langle\vec{p}, \vec{p}\rangle_{L}<0\right)$. The norm of the vector $\vec{p} \in \mathbb{R}_{1}^{3}$ is defined by $\|\vec{p}\|=\sqrt{\left|\langle\vec{p}, \vec{p}\rangle_{L}\right|}$. Lorentzian vector product $\vec{p} \times \vec{q}$ of $\vec{p}$ and $\vec{q}$ is defined as follows:

$$
\vec{p} \times \vec{q}=\left|\begin{array}{ccc}
e_{1} & e_{2} & -e_{3} \\
p_{1} & p_{2} & p_{3} \\
q_{1} & q_{2} & q_{3}
\end{array}\right|
$$


Now we define a non-degenerate rotational surface in $\mathbb{R}_{1}^{3}$. For an open interval $I \subset \mathbb{R}$, let $\gamma: I \longrightarrow \Pi$ be a curve in a plane $\Pi$ in $\mathbb{R}_{1}^{3}$, and let $\ell$ be a straight line in $\Pi$ which does not intersect the curve $\gamma$. A rotational surface in $\mathbb{R}_{1}^{3}$ is defined as a non degenerate surface rotating a curve $\gamma$ around a line $\ell$ (these are called the profile curve and the axis, respectively).

If the axis $l$ is spacelike (resp. timelike and lightlike) in Minkowski 3 -space $\mathbb{R}_{1}^{3}$, then we may suppose that $l$ is the line spanned by the vector $(1,0,0)$, (resp. $(0,0,1)$ and. $(0,1,1))$. The semi-orthogonal matrices given as follow is the subgroup of the Lorentzian group that fixes the above vectors as invariant

$$
\begin{gathered}
S(v)=\left(\begin{array}{ccc}
1 & 0 & 0 \\
0 & \cosh (v) & \sinh (v) \\
0 & \sinh (v) & \cosh (v)
\end{array}\right), T(v)=\left(\begin{array}{ccc}
\cos (v) & -\sin (v) & 0 \\
\sin (v) & \cos (v) & 0 \\
0 & 0 & 1
\end{array}\right), \\
L(v)=\left(\begin{array}{ccc}
1 & -v & v \\
v & 1-\frac{v^{2}}{2} & \frac{v^{2}}{2} \\
v & -\frac{v^{2}}{2} & 1+\frac{v^{2}}{2}
\end{array}\right), v \in \mathbb{R} .
\end{gathered}
$$

The matrices $X=\{S, T, L\}$ can be found by solving the following equations simultaneously

i. $\quad X \cdot \ell=\ell$,

ii. $X^{t} \varepsilon X=\varepsilon$ where $\epsilon=\operatorname{diag}(1,1,-1)$,

iii. $\operatorname{det} X=1$.

A rotational surface in Minkowski 3-space with the spacelike (resp. timelike and lightlike) axis which is spanned by the vector $(1,0,0)(\operatorname{resp} .(0,0,1)$ and. $(0,1,1))$ as follow

$$
R(u, v)=X(v) \cdot \gamma(u)
$$

A surface in $\mathbb{R}_{1}^{3}$ is timelike surface if the $D=E G-F^{2}<0$ where $E, F, G$ are the coefficients of the first fundamental form.

Parametrization of the profile curve $\gamma$ is given by $\gamma(u)=(f(u), g(u), h(u))$ where $f(u), g(u)$ and $h(u)$ are differentiable functions for all $u \in \mathbb{R} \backslash\{0\}$. If $\gamma(u)$ lightlike curve then $\left\langle\gamma^{\prime}(u), \gamma^{\prime}(u)>_{L}=f^{\prime 2}+g^{\prime 2}-h^{\prime 2}=0\right.$ and $h=\int \sqrt{f^{\prime 2}+g^{\prime 2}} d u$. 

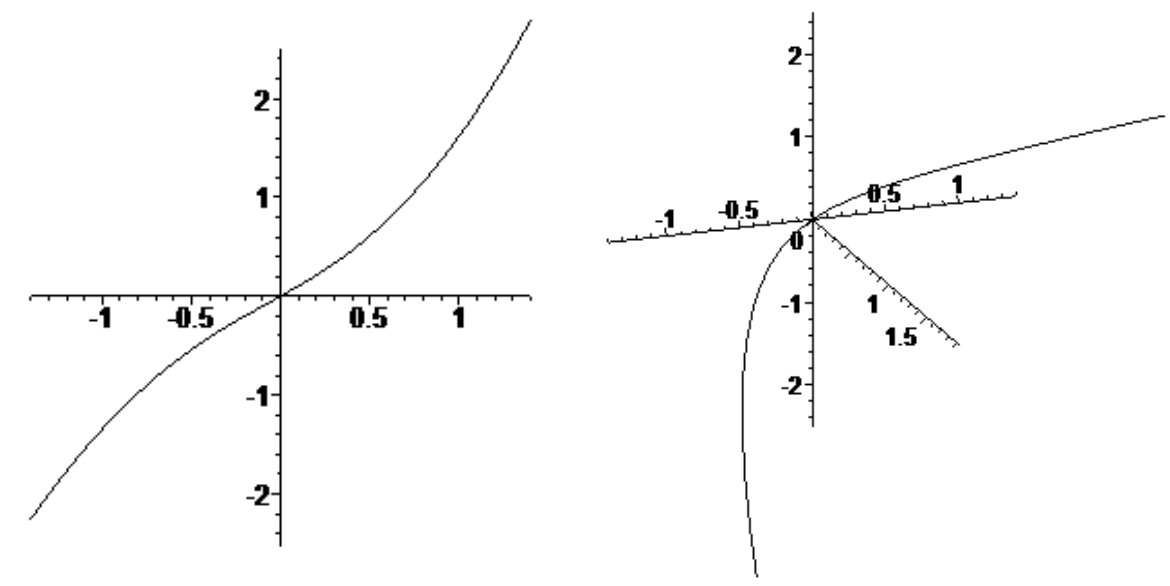

Figure 1. a-b A lightlike profile curve.

An example of a lightlike profile curve in Minkowski 3-space (see Fig.1) is given by

$$
\gamma(u)=\left(u^{2}, u, \int \sqrt{4 u^{2}+1} d u\right) .
$$

where $\int \sqrt{4 u^{2}+1} d u=\frac{1}{2} u \sqrt{4 u^{2}+1}+\frac{1}{4} \sinh ^{-1}(2 u)+c(c=$ const. $)$.

\section{TRS with LPC and the Gauss map}

We classify a rotational surface by types of axis and profile curve, and we write it as (axis's type, profile curve's type)-type; for example, $(S, L)$ - type mean that the surface has a spacelike axis and a lightlike profile curve. We give rotational surfaces with lightlike profile curve that are used to obtain the main theorems in this paper. If the profile curve, $\gamma$, is a lightlike (LPC), then the rotational surface is a timelike (TRS) with spacelike, timelike or lightlike axis and they are $(S, L)$, $(T, L)$ and $(L, L)$-types respectively.

When the axis $\ell$ is spacelike, there is a Lorentz transformation by which the axis $\ell$ is transformed to the $p_{1}$-axis of $\mathbb{R}_{1}^{3}$. If the profile curve is $\gamma(u)=\left(u^{2}, u, h(u)\right)$, then a TRS $R(u, v)$ can be written as

$$
R(u, v)=\left(\begin{array}{c}
u^{2} \\
u \cosh (v)+h(u) \sinh (v) \\
u \sinh (v)+h(u) \cosh (v)
\end{array}\right) .
$$



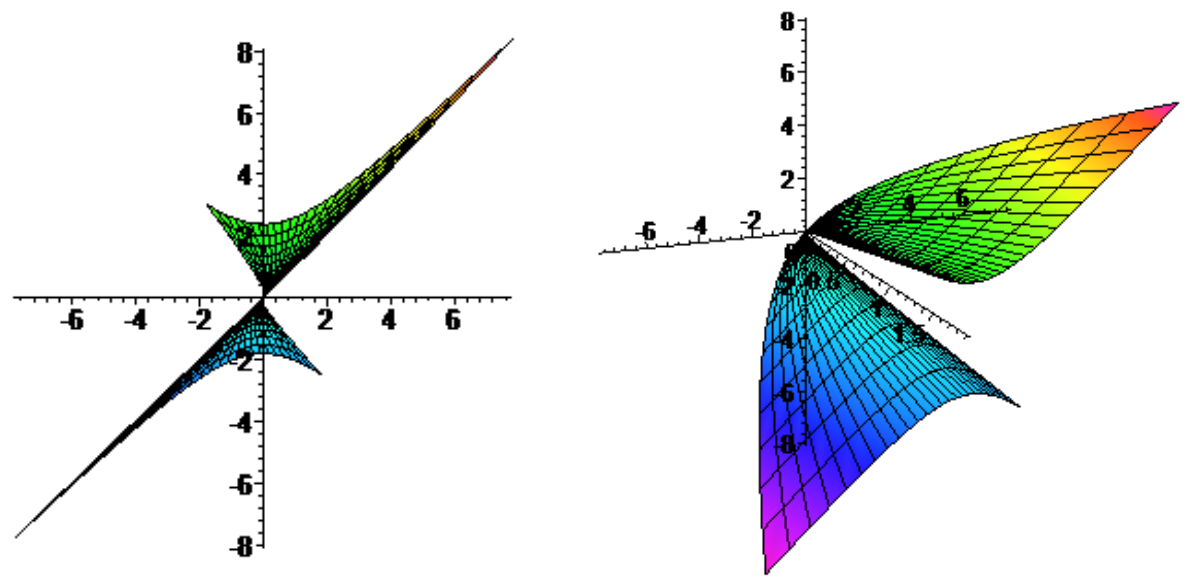

Figure 2. a-b TRS with LPC of (S,L)-type.

Proposition 1. A TRS with LPC of (S,L)-type is as follows (see Fig. 2)

$$
R(u, v)=\left(\begin{array}{c}
u^{2} \\
u \cosh (v)+\left(\frac{1}{2} u \sqrt{4 u^{2}+1}+\frac{1}{4} \sinh ^{-1}(2 u)\right) \sinh (v) \\
u \sinh (v)+\left(\frac{1}{2} u \sqrt{4 u^{2}+1}+\frac{1}{4} \sinh ^{-1}(2 u)\right) \cosh (v)
\end{array}\right)
$$

where $u, v \in \mathbb{R} \backslash\{0\}$.
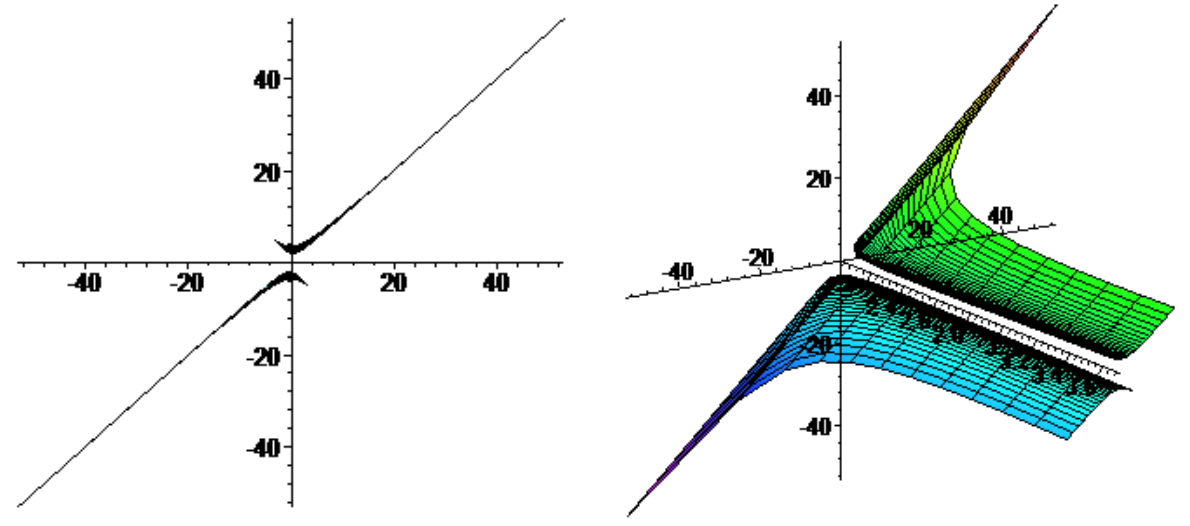

Figure 3. a-b Gauss map of the TRS with LPC of (S,L)-type. 
Proposition 2. If an (S,L)-type a TRS with LPC is as above, then the Gauss map is (see Fig. 3)

$$
\mathbf{N}=\frac{1}{\sqrt{|D|}}\left(\begin{array}{c}
u-h h^{\prime} \\
-2 u(u \cosh (v)+h \sinh (v)) \\
-2 u(u \sinh (v)+h \cosh (v))
\end{array}\right)
$$

where $D=-\left(h-u h^{\prime}\right)^{2}, h=h(u)=\frac{u \sqrt{4 u^{2}+1}}{2}+\frac{\sinh ^{-1}(2 u)}{4}, h(u)<c u, \quad c, u, v \in$ $\mathbb{R} \backslash\{0\}$.

When the axis $\ell$ is timelike, there is a Lorentz transformation by which the axis $\ell$ is transformed to the $p_{3}$-axis of $\mathbb{R}_{1}^{3}$. If the profile curve is $\gamma(u)=\left(u^{2}, u, h(u)\right)$, then a TRS $R(u, v)$ can be written as

$$
R(u, v)=\left(\begin{array}{c}
u^{2} \cos (v)-u \sin (v) \\
u^{2} \sin (v)+u \cos (v) \\
h(u)
\end{array}\right) .
$$
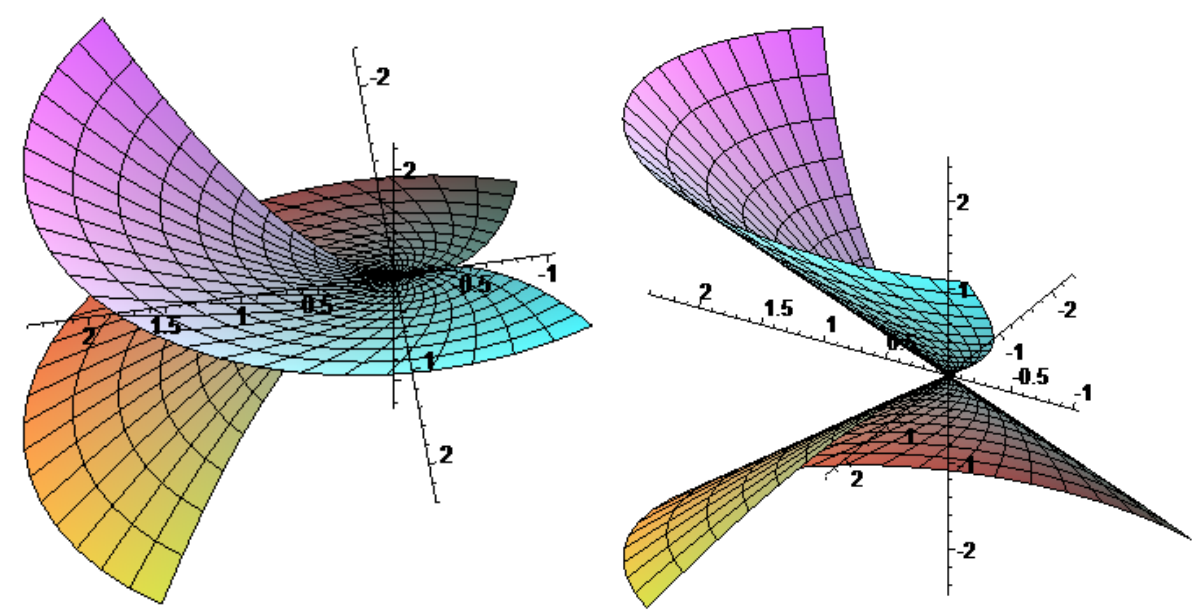

Figure 4. a-b TRS with LPC of (T,L)-type.

Proposition 3. A TRS with LPC of (T,L)-type is as follows (see Fig. 4)

$$
R(u, v)=\left(\begin{array}{c}
u^{2} \cos (v)-u \sin (v) \\
u^{2} \sin (v)+u \cos (v) \\
\frac{1}{2} u \sqrt{4 u^{2}+1}+\frac{1}{4} \sinh ^{-1}(2 u)
\end{array}\right)
$$

where $u, v \in \mathbb{R} \backslash\{0\}$. 
Proposition 4. If an (T,L)-type a TRS with LPC is as above, then the Gauss map is (see Fig. 5)

$$
\mathbf{N}=\frac{1}{\sqrt{|D|}}\left(\begin{array}{c}
-u h^{\prime} \sin (v)-u^{2} h^{\prime} \cos (v) \\
-u h^{\prime} \cos (v)-u^{2} h^{\prime} \sin (v) \\
-2 u^{3}+u
\end{array}\right)
$$

where $D=-u^{4}, h=h(u)=\frac{u \sqrt{4 u^{2}+1}}{2}+\frac{\sinh ^{-1}(2 u)}{4}, u, v \in \mathbb{R} \backslash\{0\}$.
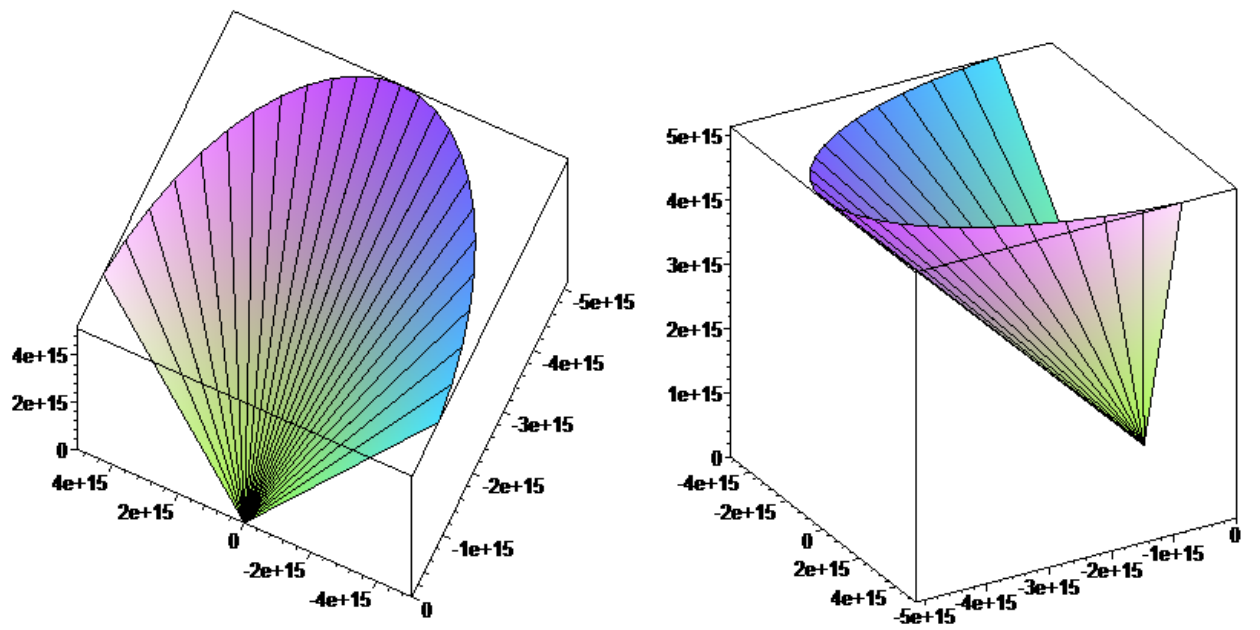

Figure 5. a-b Gauss map of a TRS with LPC of (T,L)-type.

When the axis $\ell$ is lightlike, there is a Lorentz transformation by which the axis $\ell$ is transformed into the $p_{2} p_{3}$-axis of $\mathbb{R}_{1}^{3}$. If the profile curve is $\gamma(u)=\left(u^{2}, u, h(u)\right)$, then a TRS $R(u, v)$ can be written as

$$
R(u, v)=\left(\begin{array}{c}
u^{2}-u v+h v \\
u^{2} v+\left(1-\frac{v^{2}}{2}\right) u+\frac{v^{2}}{2} h \\
u^{2} v-\frac{v^{2}}{2} u+\left(1+\frac{v^{2}}{2}\right) h
\end{array}\right) .
$$

Proposition 5. A TRS with LPC of (L,L)-type is as follows (see Fig. 6)

$$
R(u, v)=\left(\begin{array}{c}
u^{2}-u v+\frac{u v \sqrt{4 u^{2}+1}}{2}+\frac{v \sinh ^{-1}(2 u)}{4} \\
u^{2} v+\left(1-\frac{v^{2}}{2}\right) u+\frac{u v^{2} \sqrt{4 u^{2}+1}}{4}+\frac{v^{2} \sinh ^{-1}(2 u)}{8} \\
u^{2} v-\frac{v^{2}}{2} u+\left(1+\frac{v^{2}}{2}\right)\left(\frac{u \sqrt{4 u^{2}+1}}{2}+\frac{\sinh ^{-1}(2 u)}{4}\right)
\end{array}\right)
$$

where $u, v \in \mathbb{R} \backslash\{0\}$. 

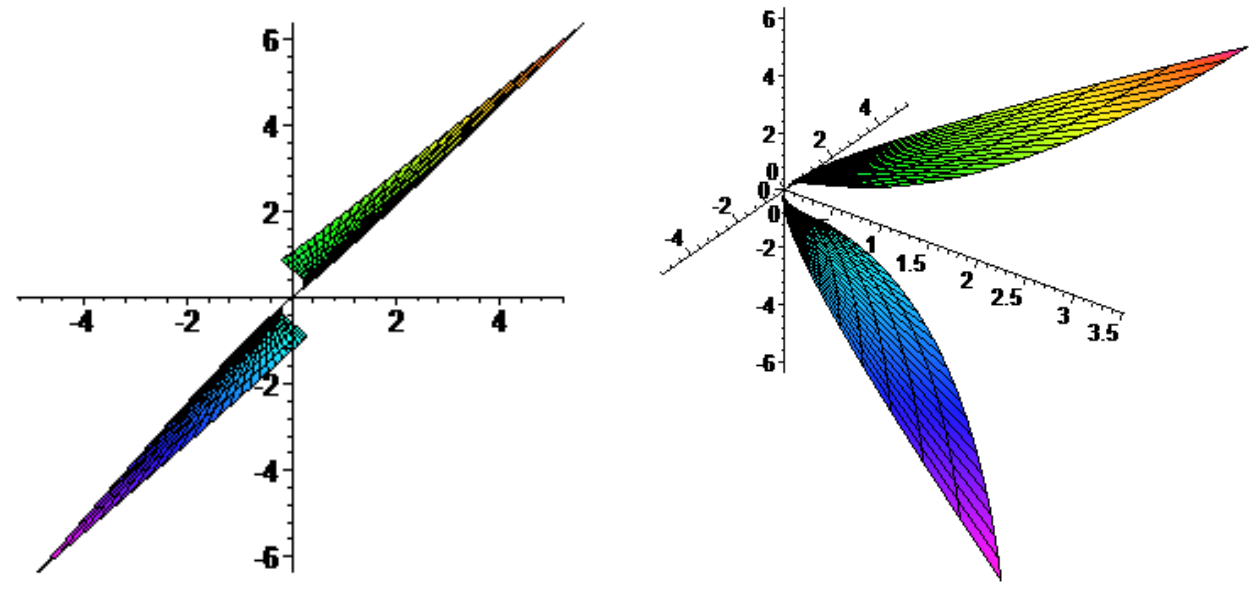

Figure 6. a-b TRS with LPC of (L,L)-type.

Proposition 6. If an (L,L)-type TRS with LPC is as above, then the Gauss map is (see Fig. 7)

$$
\mathbf{N}=\frac{1}{\sqrt{|D|}}\left(\begin{array}{c}
\left(-u^{2}+u v-h v\right) h^{\prime}+v h+\left(u^{2}-u v\right) \\
\left(-u^{2} v+\frac{v^{2}}{2} h-u+h\right) h^{\prime}+\frac{v^{2}}{2} h-2 u^{3}+u^{2} v-\frac{u v^{2}}{2} \\
\left(-u^{2} v+\frac{v^{2}}{2} h-v^{2}\right) h^{\prime}+\left(\frac{v^{2}}{2}-1\right) h-2 u^{3}+u^{2} v-\frac{u v^{2}}{2}
\end{array}\right)
$$

where $D=-\left(-u^{2} h^{\prime}+2 u h-u^{2}\right)^{2}, h=h(u)=\frac{u \sqrt{4 u^{2}+1}}{2}+\frac{\sinh ^{-1}(2 u)}{4}, h(u)<u^{2}+u$, $u, v \in \mathbb{R} \backslash\{0\}$. 

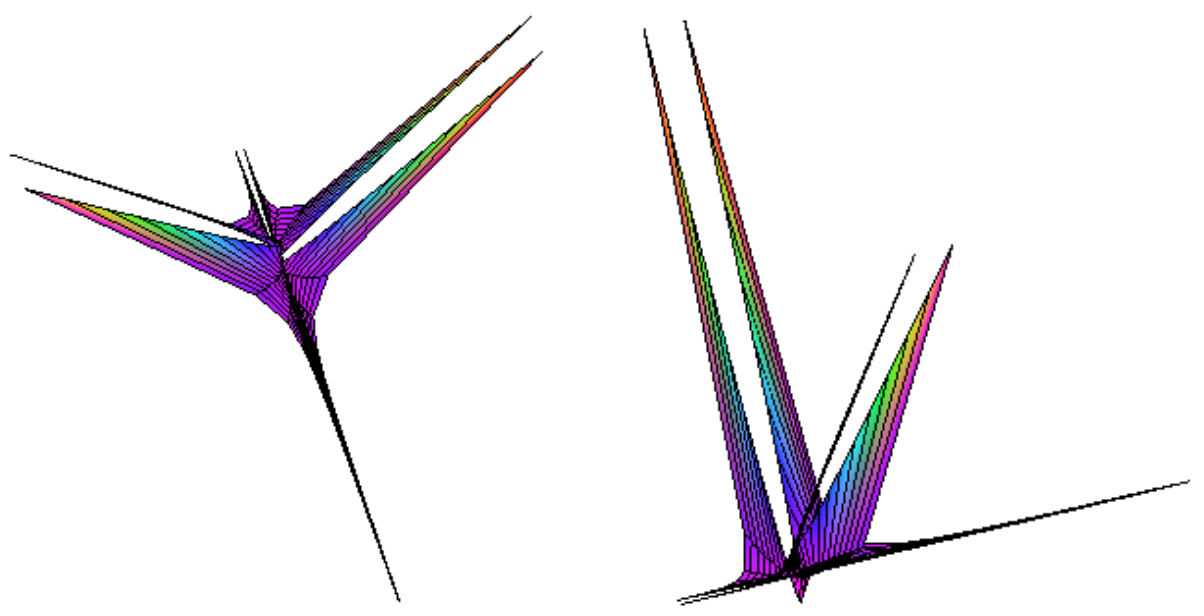

Figure 7. a-b Gauss map of a TRS with LPC of (L,L)-type.

\section{Curvatures and Laplacian}

In this section, we study mean curvature, Gaussian curvature and LaplaceBeltrami operators of the geometric objects of a TRS with LPC as (axis's type, profile curve's type)-type.

Case 1. A timelike rotational surface of $(S, L)$ - type.

In this case, we assume that the axis $l$ is spacelike $(1,0,0)$ vector, $\gamma(u)=$ $\left(u^{2}, u, h(u)\right)$ profile curve is a lightlike curve and $h=h(u)=2^{-1} u \sqrt{4 u^{2}+1}+$ $2^{-2} \sinh ^{-1}(2 u), u \in \mathbb{R} \backslash\{0\}$.

Theorem 4.1. The mean curvature and the Gaussian curvature of a TRS with $L P C$ of $(S, L)$-type are related as

$$
H^{2}=\varrho \cdot K
$$

in Minkowski 3-space, where

$$
\begin{aligned}
& \varrho(u)=-4 u^{-2}\left[-2 u\left(h-u h^{\prime}\right)^{2}-\left(-u^{2}+h^{2}\right)\left(u-h h^{\prime}+u h^{\prime} h^{\prime \prime}\right)\right]^{2}, \\
& D=-\left(h-u h^{\prime}\right)^{2}, h(u)=\frac{u \sqrt{4 u^{2}+1}}{2}+\frac{\sinh ^{-1}(2 u)}{4} \\
& \text { and } \\
& u \in \mathbb{R} \backslash\{0\} .
\end{aligned}
$$

Proof. We consider a rotational surface (4). Components of the first fundamental form are

$$
E=0, \quad F=h-u h^{\prime}, \quad G=-u^{2}+h^{2}
$$


and the second fundamental form are

$$
e=\frac{2\left(u-h h^{\prime}+u h^{\prime} h^{\prime \prime}\right)}{\sqrt{\left(h-u h^{\prime}\right)^{2}}}, \quad f=\frac{-2 u\left(u h^{\prime}-h\right)}{\sqrt{\left(h-u h^{\prime}\right)^{2}}}, g=0 .
$$

Since $D=-\left(h-u h^{\prime}\right)^{2}<0$ then $R(u, v)$ is a timelike surface. The mean curvature and the Gaussian curvature of a TRS with LPC of $(S, L)$-type are given as follows, respectively,

$$
\begin{gathered}
H=\frac{\left[-2 u\left(h-u h^{\prime}\right)^{2}-\left(-u^{2}+h^{2}\right)\left(u-h h^{\prime}+u h^{\prime} h^{\prime \prime}\right)\right]^{2}}{\left(h-u h^{\prime}\right)^{3 / 2}}, \\
K=\frac{-4 u^{2}}{\left(h-u h^{\prime}\right)^{2}}
\end{gathered}
$$

where $u \in \mathbb{R} \backslash\{0\}$. Hence, we get the results.

Theorem 4.2. The Gauss map and its Laplacian of a TRS with LPC of $(S, L)$-type are related as

$$
\Delta \mathbf{N}=\partial+\mathbf{N}
$$

in Minkowski 3-space, where $\mathbf{N}=\mathbf{N}(u, v)=D^{-1 / 2}\left(\mathbf{N}_{\mathbf{1}} \mathbf{N}_{\mathbf{2}}, \mathbf{N}_{\mathbf{3}}\right)$ in (6),

$$
\begin{aligned}
& \partial=\partial(u, v)=-\frac{1}{4(D)^{7 / 2}}\left(\begin{array}{l}
\partial_{1}(u, v) \\
\partial_{2}(u, v) \\
\partial_{3}(u, v)
\end{array}\right), \\
& \partial_{1}(u, v)=\left(2 D G_{u}-F_{u} G\right)\left[2 D\left(\mathbf{N}_{1}\right)_{u}-\mathbf{N}_{1} D_{u}\right] \\
& +\left\{\left[D\left(\mathbf{N}_{\mathbf{1}}\right)_{u u}-D_{u}\left(\mathbf{N}_{\mathbf{1}}\right)_{u}\right] 4 D-2 \mathbf{N}_{\mathbf{1}} D D_{u u}-\mathbf{N}_{\mathbf{1}} D_{u}^{2}\right\} G+4 D^{3} \mathbf{N}_{\mathbf{1}} \text {, } \\
& \mathrm{\partial}_{2}(u, v)=\left(2 D G_{u}-F_{u} G\right)\left[2 D\left(\mathbf{N}_{\mathbf{2}}\right)_{u}-\mathbf{N}_{\mathbf{2}} D_{u}\right] \\
& +\left\{\left[D\left(\mathbf{N}_{\mathbf{2}}\right)_{u u}-D_{u}\left(\mathbf{N}_{\mathbf{2}}\right)_{u}\right] 4 D-2 \mathbf{N}_{\mathbf{2}} D D_{u u}-\mathbf{N}_{\mathbf{2}} D_{u}^{2}\right\} G \\
& -2\left[2\left(\mathbf{N}_{\mathbf{2}}\right)_{u v} D-\left(\mathbf{N}_{\mathbf{2}}\right)_{v} D_{u}\right] D^{1 / 2}+4 D^{3} \mathbf{N}_{\mathbf{2}} \text {, } \\
& ð_{3}(u, v)=\left(2 D G_{u}-F_{u} G\right)\left[2 D\left(\mathbf{N}_{\mathbf{3}}\right)_{u}-\mathbf{N}_{\mathbf{3}} D_{u}\right] \\
& +\left\{\left[D\left(\mathbf{N}_{\mathbf{3}}\right)_{u u}-D_{u}\left(\mathbf{N}_{\mathbf{3}}\right)_{u}\right] 4 D-2 \mathbf{N}_{\mathbf{3}} D D_{u u}-\mathbf{N}_{\mathbf{3}} D_{u}^{2}\right\} G \\
& -2\left[2\left(\mathbf{N}_{\mathbf{3}}\right)_{u v} D-\left(\mathbf{N}_{\mathbf{3}}\right)_{v} D_{u}\right] D^{1 / 2}+4 D^{3} \mathbf{N}_{\mathbf{3}} \\
& D=-\left(h-u h^{\prime}\right)^{2}, \\
& F=h-u h^{\prime} \text {, } \\
& G=-u^{2}+h^{2} \text {, } \\
& u, v \in \mathbb{R} \backslash\{0\} .
\end{aligned}
$$

Proof. We use the Gauss map of a TRS with LPC of $(S, L)$-type in (6).Hence,

$$
\mathbf{N}_{u}=\frac{1}{2 D^{3 / 2}}\left(\begin{array}{c}
2 D\left(\mathbf{N}_{\mathbf{1}}\right)_{u}-D_{u} \mathbf{N}_{\mathbf{1}} \\
2 D\left(\mathbf{N}_{\mathbf{2}}\right)_{u}-D_{u} \mathbf{N}_{\mathbf{2}} \\
2 D\left(\mathbf{N}_{\mathbf{3}}\right)_{u}-D_{u} \mathbf{N}_{\mathbf{3}}
\end{array}\right), \mathbf{N}_{v}=\frac{1}{\sqrt{|D|}}\left(\begin{array}{c}
0 \\
-2 u(u \sinh (v)+h \cosh (v)) \\
-2 u(u \cosh (v)+h \sinh (v))
\end{array}\right)
$$




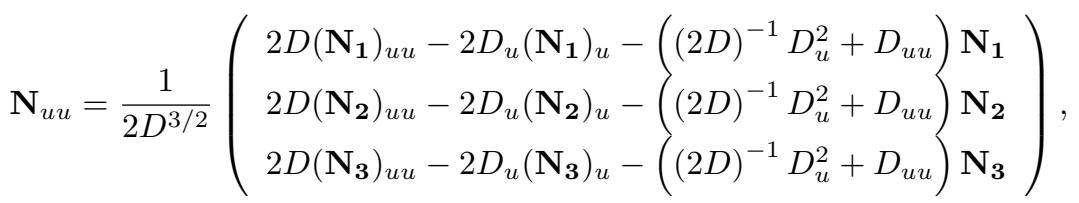

$$
\begin{aligned}
& \mathbf{N}_{u v}=\frac{1}{2 D^{3 / 2}}\left(\begin{array}{c}
0 \\
2 D\left(\mathbf{N}_{2}\right)_{u v}-D_{u}\left(\mathbf{N}_{2}\right)_{v} \\
2 D\left(\mathbf{N}_{3}\right)_{u v}-D_{u}\left(\mathbf{N}_{3}\right)_{v}
\end{array}\right) \\
& \mathbf{N}_{v v}=\frac{1}{\sqrt{|D|}}\left(\begin{array}{c}
0 \\
-2 u(u \cosh (v)+h \sinh (v)) \\
-2 u(u \sinh (v)+h \cosh (v))
\end{array}\right) \text {. }
\end{aligned}
$$

Therefore, from (1) we get

$$
\Delta \mathbf{N}=-\frac{\left\{2\left[\left(G_{u}-F_{v}\right) \mathbf{N}_{u}-F_{u} \mathbf{N}_{v}+G \mathbf{N}_{u u}-2 F \mathbf{N}_{u v}\right] D-\left(G \mathbf{N}_{u}-F \mathbf{N}_{v}\right) D_{u}+F D_{v} \mathbf{N}_{u}\right\}}{2 D^{2}}
$$

where $D=-\left(h-u h^{\prime}\right)^{2}$ and $u, v \in R \backslash\{0\}$. Using (1), (2) and (6), we can see the components of the Laplacian of the Gauss map $\left(\Delta \mathbf{N}_{1}, \Delta \mathbf{N}_{2}, \Delta \mathbf{N}_{3}\right)$ of a TRS with LPC of $(S, L)$-type.

Theorem 4.3. A TRS with LPC of $(S, L)$-type and its Laplacian are related as (see Fig. 8)

$$
\Delta R=\boldsymbol{\epsilon}+R
$$

in Minkowski 3-space, where $R=R(u, v)$ in (4),

$$
\begin{aligned}
& \boldsymbol{\epsilon}=\boldsymbol{\epsilon}(u, v)=\frac{1}{\left(h-u h^{\prime}\right)^{3}}\left(\begin{array}{c}
\mathbf{k}_{1}(u) \\
\mathbf{k}_{2}(u) \cosh (v)+\mathbf{k}_{3}(u) \sinh (v) \\
\mathbf{k}_{2}(u) \sinh (v)+\mathbf{k}_{3}(u) \cosh (v)
\end{array}\right), \\
& \mathbf{k}_{1}(u)=\quad-4 u^{2} h-u^{2} h^{3}+u\left(4 u^{2}+4 h^{2}-3 u^{2} h^{2}\right) h^{\prime} \\
&+u^{2}\left(-4-3 u^{2}\right) h h^{\prime 2}+u^{5} h^{\prime 3}-2 u^{2}\left(u^{2}-h^{2}\right) h^{\prime \prime}, \\
& \mathbf{k}_{2}(u)=\quad-2 u h-u^{3} h^{3}+\left(2 u^{2}+2 h^{2}-u^{2} h+h^{3}-2 h+3 u^{4} h^{2}\right) h^{\prime} \\
&+\left(-2 u h+u^{3}-u h^{2}+2 u-3 u^{5} h\right) h^{\prime 2}+u^{6} h^{\prime 3}-u\left(u^{2}-h^{2}\right) h^{\prime \prime}, \\
& \mathbf{k}_{3}(u)=\quad-\left(u^{2}-2\right) h+h^{3}-u^{2} h^{4}+u\left(u^{2}-h^{2}+2-2 h+3 u^{2} h^{3}\right) h^{\prime}, \\
&+\left(2 u^{2}+2 h^{2}-3 u^{4} h^{2}\right) h^{\prime 2}+u\left(-2+u^{4}\right) h h^{\prime 3}-u\left(u^{2}-h^{2}\right) h^{\prime \prime}, \\
& \text { and } \quad \frac{u \sqrt{4 u^{2}+1}}{2}+\frac{\sinh ^{-1}(2 u)}{4}, u, v \in \mathbb{R} \backslash\{0\} .
\end{aligned}
$$



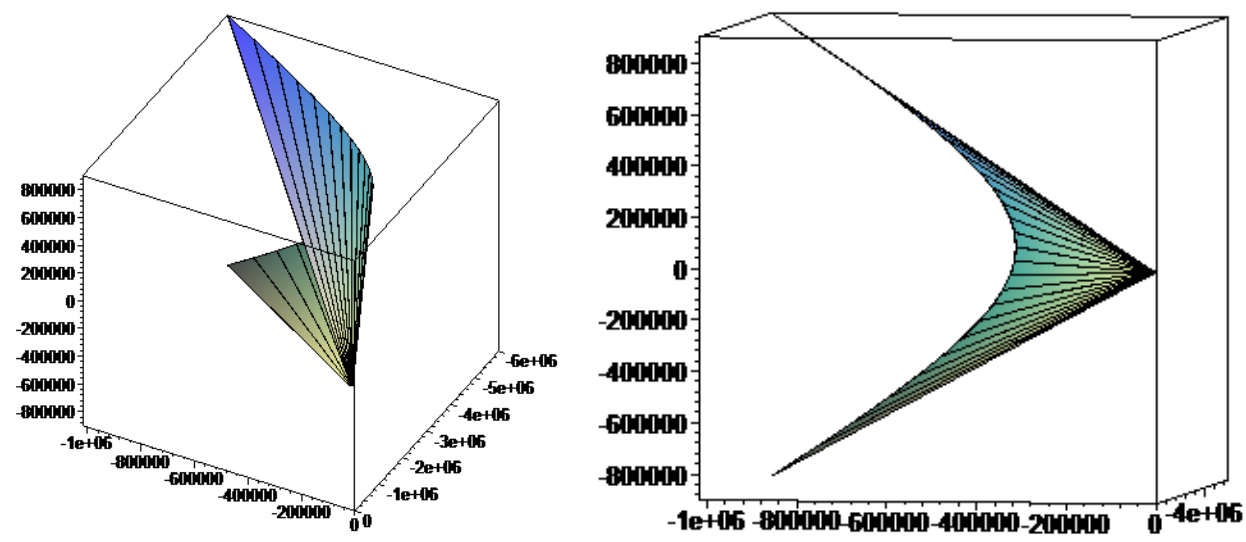

Figure 8. a-b $\Delta R$ of a TRS with LPC of (S,L)-type.

Proof. Using (1), (2) and (4), we get $\left(\Delta R_{1}, \Delta R_{2}, \Delta R_{3}\right)$ easily.

Case 2. A timelike rotational surface of $(T, L)-$ type.

In this case, we assume that the axis $l$ is timelike $(0,0,1)$ vector, $\gamma(u)=\left(u^{2}, u, h(u)\right)$ profile curve is a lightlike curve and $h(u)=2^{-1} u \sqrt{4 u^{2}+1}+2^{-2} \sinh ^{-1}(2 u), u \in \mathbb{R} \backslash\{0\}$.

Theorem 4.4. The mean curvature and the Gausssian curvature of a TRS with $L P C$ of $(T, L)$-type are related as

$$
H^{2}=\Upsilon \cdot K
$$

in Minkowski 3-space, where

$$
\begin{aligned}
& \Upsilon= \Upsilon(u, v)=\frac{\chi^{2}}{\vartheta} \\
& \chi= \chi(u, v)=\left[2\left(u^{3}+u-1\right) \sin (v) \cos (v)+4 u \cos ^{2}(v)+u^{4}+u^{2}-3 u\right] h^{\prime}, \\
& \vartheta= \vartheta(u, v)=\left\{\left[2 u \cos ^{2}(v)-4 \cos ^{2}(v)+2 u^{3}-u+3\right] 4 u \sin (v) \cos (v)\right. \\
&+\left[4 u^{2} \cos ^{2}(v)-2 u^{3} \cos ^{2}(v)-\cos ^{2}(v)+3 u^{3}-6 u^{2}+1\right] 4 \cos ^{2}(v) \\
&\left.+2 u^{5}-2 u^{3}+9 u^{2}\right\} h^{\prime 2}, \\
& D=-u^{4} \\
& \text { and } \vartheta(u, v) \neq 0, u, v \in \mathbb{R} \backslash\{0\} .
\end{aligned}
$$

Proof. We consider a rotational surface (7). Components of the first and second fundamental forms are given as follows, respectively,

$$
E=0, \quad F=-u^{2}, \quad G=u^{4}+u^{2},
$$


and

$$
\begin{gathered}
e=(-4 \sin (v) \cos (v)+2 u) u^{-1} h^{\prime}, \\
f=\left[-2 u\left(\cos ^{2}(v)-\sin ^{2}(v)\right)+2 \sin (v) \cos (v)+u\right] u^{-1} h^{\prime}
\end{gathered}
$$

$$
g=\left[\left(\cos ^{2}(v)-\sin ^{2}(v)\right) u+2 u^{2} \sin (v) \cos (v)+u^{3}\right] u^{-1} h^{\prime} .
$$

Since $D=-u^{4}<0$ then $R(u, v)$ is a timelike surface. Then, the mean curvature and the Gaussian curvature of a TRS with LPC of $(T, L)$-type are

$$
\begin{gathered}
H=\frac{\chi(u, v)}{u^{4}}, \\
K=\frac{\vartheta(u, v)}{u^{8}}
\end{gathered}
$$

where

$$
\begin{aligned}
\chi(u, v)= & {\left[2\left(u^{3}+u-1\right) \sin (v) \cos (v)+4 u \cos ^{2}(v)+u^{4}+u^{2}-3 u\right] h^{\prime}, } \\
\vartheta(u, v)= & \left\{4 u\left[2 u \cos ^{2}(v)-4 \cos ^{2}(v)+2 u^{3}-u+3\right] \sin (v) \cos (v)\right. \\
& +4\left[4 u^{2} \cos ^{2}(v)-2 u^{3} \cos ^{2}(v)-\cos ^{2}(v)+3 u^{3}-6 u^{2}+1\right] \cos ^{2}(v) \\
& \left.+2 u^{5}-2 u^{3}+9 u^{2}\right\} h^{\prime 2},
\end{aligned}
$$

$u, v \in \mathbb{R} \backslash\{0\}$. Then we can easily see the results.

Theorem 4.5. The Gauss map and its Laplacian of a TRS with LPC of $(T, L)$-type are related as (see Fig. 9)

$$
\Delta \mathbf{N}=\Theta+\mathbf{N}
$$

in Minkowski 3-space, where $\mathbf{N}=\mathbf{N}(u, v)$ in (9),

$$
\begin{aligned}
\Theta=\Theta(u, v)=\frac{1}{|D|}\left(\begin{array}{c}
\varphi_{1}(u) \sin (v)+\varphi_{2}(u) \cos (v) \\
\sigma_{1}(u) \cos (v)+\sigma_{2}(u) \sin (v) \\
2 u^{5}+2 u^{4}+9 u^{3}+3 u^{2}+8 u+1
\end{array}\right), & \\
\varphi_{1}(u)=\quad & \left(u^{3}+u\right) h^{\prime \prime \prime}+\left(-u^{3}-2 u^{2}+3 u\right) h^{\prime \prime} \\
& +\left(-2 u^{5}+u^{3}+u^{2}+4 u+1\right) h^{\prime}, \\
\varphi_{2}(u)= & \left(-u^{5}-u^{3}\right) h^{\prime \prime \prime}+\left(-u^{5}-5 u^{4}-u^{3}-3 u^{2}-4 u\right) h^{\prime \prime} \\
& +\left(2 u^{5}+u^{4}-2 u+4\right) h^{\prime}, \\
\sigma_{1}(u)= & \left(u^{3}+u\right) h^{\prime \prime \prime}+\left(-u^{3}-2 u^{2}-5 u\right) h^{\prime \prime} \\
& +\left(2 u^{5}+u^{3}+u^{2}+1\right) h^{\prime}, \\
\sigma_{2}(u)= & \left(-u^{5}-u^{3}\right) h^{\prime \prime \prime}+\left(-u^{5}-5 u^{4}-u^{3}-3 u^{2}+4 u\right) h^{\prime \prime} \\
& +\left(-2 u^{5}+u^{4}+2 u-4\right) h^{\prime}, \\
& -u^{4} \\
D= &
\end{aligned}
$$


Proof. We assume that the profile curve is $\gamma(u)=\left(u^{2}, u, h(u)\right)$. Using (1), (2) and (9), we get the components of the Laplacian of the Gauss map of a TRS with LPC of $(T, L)$-type are

$$
\begin{aligned}
\Delta \mathbf{N}_{1}= & {\left[\left(u+u^{-1}\right) h^{\prime \prime \prime}+\left(-u-2+3 u^{-1}\right) h^{\prime \prime}\right.} \\
& \left.+\left(-2 u^{3}+1+4 u^{-1}+u^{-2}\right) h^{\prime}\right] \sin (v) \\
& +\left[\left(-u^{3}-u\right) h^{\prime \prime \prime}+\left(-u^{3}-5 u^{2}-u-3-4 u^{-1}\right) h^{\prime \prime}\right. \\
& \left.+2\left(u^{3}-u^{-1}+2 u^{-2}\right) h^{\prime}\right] \cos (v), \\
\Delta \mathbf{N}_{2}= & {\left[\left(u+u^{-1}\right) h^{\prime \prime \prime}+\left(-u-2-5 u^{-1}\right) h^{\prime \prime}\right.} \\
& \left.+\left(2 u^{3}+1+u^{-2}\right) h^{\prime}\right] \cos (v) \\
& +\left[\left(-u^{3}-u\right) h^{\prime \prime \prime}+\left(-u^{3}-5 u^{2}-u-3+4 u^{-1}\right) h^{\prime \prime}\right. \\
& \left.+2\left(-u^{3}+u^{-1}-2 u^{-2}\right) h^{\prime}\right] \sin (v) \\
\text { and } & \\
\Delta \mathbf{N}_{3}= & 2 u^{2}+10 u+3+8 u^{-1}+u^{-2}, u, v \in \mathbb{R} \backslash\{0\} .
\end{aligned}
$$

Then, we can easily see the results.
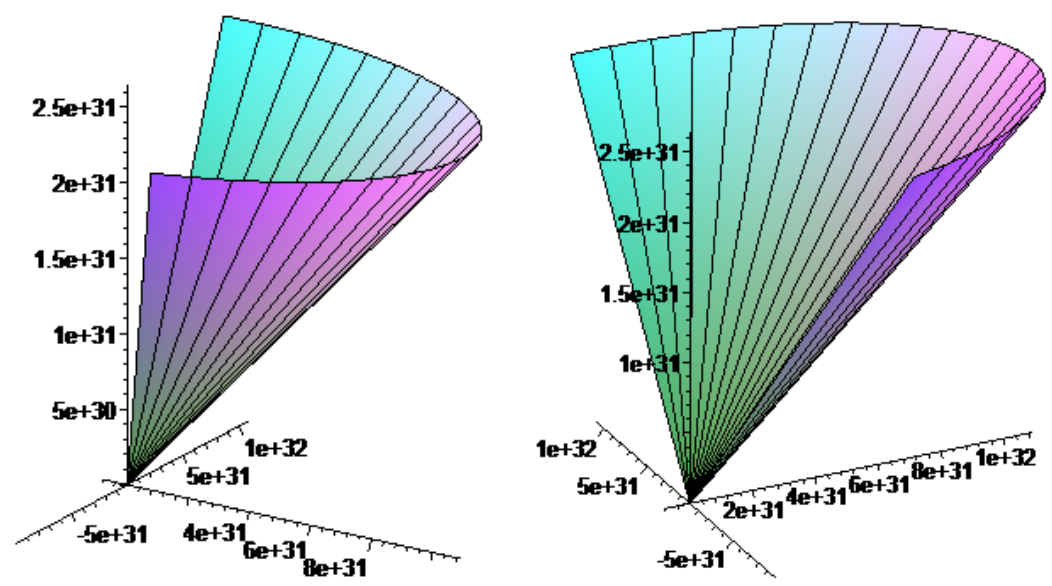

Figure 9. a-b $\Delta \mathbf{N}$ of a TRS with LPC of (T,L)-type.

Theorem 4.6. A TRS with LPC of $(T, L)$-type and its Laplacian are related as (see Fig. 10)

$$
\Delta R=\Omega+R
$$

in Minkowski 3-space, where $R=R(u, v)$ in $(7)$,

$$
\Omega=\Omega(u, v)=\left(\begin{array}{c}
\left(6+\frac{3}{u^{2}}-u^{2}\right) \cos (v)+u \sin (v) \\
\left.6+\frac{3}{u^{2}}-u^{2}\right) \sin (v)-u \cos (v) \\
\left(1+\frac{1}{u^{2}}\right) h^{\prime \prime}+\frac{2}{u} h^{\prime}-h
\end{array}\right),
$$


$h(u)=\frac{u \sqrt{4 u^{2}+1}}{2}+\frac{\sinh ^{-1}(2 u)}{4}, u, v \in \mathbb{R} \backslash\{0\}$.
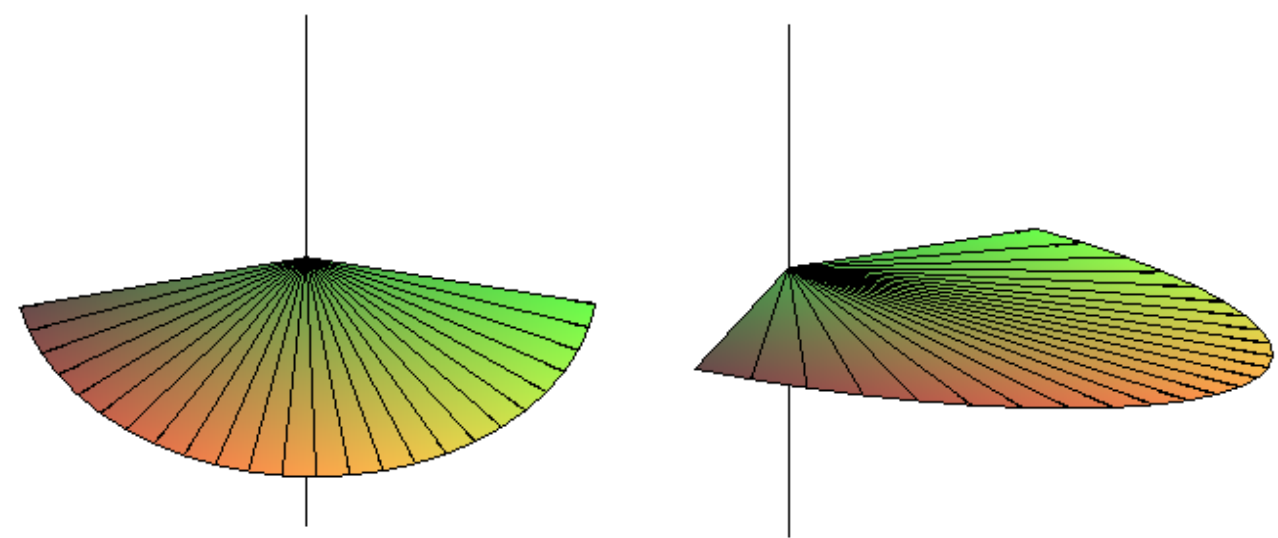

Figure 10. a-b $\Delta R$ of a TRS with LPC of (T,L)-type.

Proof. Using (1), (2) and (7) then, we get $\Delta R_{1}=3\left(2+u^{-2}\right) \cos (v), \Delta R_{2}=3$ $\left(2+u^{-2}\right) \sin (v), \Delta R_{3}=\left(1+u^{-2}\right) h^{\prime \prime}+2 u^{-1} h^{\prime}$ where $u, v \in \mathbb{R} \backslash\{0\}$.

Case 3. A timelike rotational surface of $(L, L)-$ type.

In this case, we assume that the axis $l$ is lightlike $(0,1,1)$ vector, $\gamma(u)=\left(u^{2}, u, h(u)\right)$ profile curve is a lightlike curve and $h(u)=2^{-1} u \sqrt{4 u^{2}+1}+2^{-2} \sinh ^{-1}(2 u), u \in \mathbb{R} \backslash\{0\}$.

Theorem 4.7. The mean curvature and the Gaussian curvature of a TRS with $L P C$ of $(L, L)$-type are related as

$$
H^{2}=\Psi \cdot K
$$

in Minkowski 3-space, where

$$
\Psi=\Psi(u, v)=\frac{\varsigma^{2}(u, v)}{4 D \xi(u, v)}
$$

and 


$$
\begin{aligned}
\varsigma(u, v)= & \left(h^{3}+u^{2} h+4 u^{3} v h+u^{4} v h+u^{4} v h^{\prime}-2 u^{3} v h h^{\prime}-u v^{4} h h^{\prime}\right. \\
& +\frac{3}{2} u^{3} v^{2}-3 u^{2} v^{2} h+\frac{3}{2} u v^{2} h^{2}-2 u^{2} v h^{2}-\frac{1}{2} u^{3} v^{2} h \\
& -2 u^{3} v h^{2}-\frac{1}{2} u v^{2} h^{3}+u^{2} v h^{3}+2 v^{2} h^{3} h^{\prime}+\frac{1}{2} v^{4} h^{2} h^{\prime}-\frac{3}{2} u^{3} v^{2} h^{\prime} \\
& +\frac{1}{2} u^{2} v^{4} h^{\prime}+5 u^{2} v^{2} h h^{\prime}-\frac{11}{2} u v^{2} h^{2} h^{\prime}+u^{2} v h^{2} h^{\prime}-2 u h^{2}-2 u^{4} v \\
& \left.+u^{2} v^{2} h^{2}\right) h^{\prime \prime}+\left(-4 u^{4}+12 u^{3} h+4 u^{2} v h-8 u v h^{2}-10 u^{2} h^{2}\right. \\
& +4 u^{3} h h^{\prime}+2 v^{3} h^{2}-2 u^{3} v h^{\prime}+4 u^{3} v^{2}+4 u^{2} v h h^{\prime}-8 u^{2} v^{2} h \\
& -2 u^{4} h^{\prime}+4 u^{3} h h^{\prime}-4 u v^{3} h h^{\prime}-2 u^{4} h^{\prime 2}-2 u^{2} v^{3} h^{\prime}+4 u^{3} v^{2} h^{\prime} \\
& \left.+2 u^{2} v^{3} h^{\prime}+2 u^{2} v^{3} h^{\prime 2}\right) h^{\prime}+\left(8 u^{3}-4 u^{4}+4 v h^{2}-2 u v h\right. \\
& \left.-8 u^{2} h+2 u^{3} h\right) h+2 u^{5}-2 u^{4},
\end{aligned}
$$

$$
\begin{aligned}
\xi(u, v)= & \left(h^{3}-u h^{2}+2 u^{3} v h+h^{3} h^{\prime}+u^{2} h h^{\prime}-2 u h^{2} h^{\prime}-2 u^{4} v h^{\prime}\right. \\
& -u v^{2} h h^{\prime}+3 u^{3} v h h^{\prime}+u v^{4} h h^{\prime}+u^{4} v h h^{\prime}-\frac{3}{2} u^{2} v^{2} h \\
& +\frac{3}{2} u v^{2} h^{2}-2 u^{2} v h^{2}-u^{3} v h^{2}-\frac{1}{2} u v^{2} h^{3}+u^{2} v h^{3}+v^{2} h^{2} h h^{\prime} \\
& +2 v^{2} h^{3} h^{\prime}+\frac{1}{2} h^{2} v^{4} h^{\prime}+\frac{1}{2} v^{6} h h^{\prime 2}+\frac{3}{2} u^{3} v^{2} h^{\prime}-\frac{3}{2} u^{2} v^{4} h^{\prime} \\
& +2 u^{3} v^{3} h^{\prime}+u^{4} v h^{\prime 2}-\frac{1}{2} u v^{6} h^{\prime 2}-\frac{3}{2} u^{2} v^{2} h h^{\prime}-2 u v^{2} h^{2} h^{\prime} \\
& -u^{2} v h^{2} h^{\prime}-2 u^{2} v^{3} h h^{\prime}-2 u^{3} v h h^{\prime 2}-\frac{1}{2} u^{3} v^{2} h h^{\prime}-2 u^{3} v h^{2} h^{\prime} \\
& -\frac{1}{2} u v^{2} h^{3} h^{\prime}+u^{2} v h^{3} h^{\prime}-\frac{9}{2} u v^{4} h h^{\prime 2}+\frac{1}{2} u^{2} v^{4} h h^{\prime}-u^{3} v^{3} h h^{\prime} \\
& -\frac{1}{2} u v^{4} h^{2} h^{\prime}+\frac{1}{2} u^{2} v^{2} h^{2}+2 v^{2} h^{3} h^{\prime 2}+\frac{5}{2} v^{4} h^{2} h^{\prime 2}-\frac{3}{2} u^{3} v^{2} h^{\prime 2} \\
+ & 2\left(2 v h^{2}-u^{3}+u^{2} v-3 u v h+u^{2} h+2 u^{3}+2 u v^{2}-4 u^{2} v+4 u v h\right. \\
& \left.-2 u^{2} h-2 v^{2} h-2 v^{2} h\right) h-u^{4}+2 u^{3} v-u^{2} v^{2} \\
+ & 2 u^{2} v^{4} h^{\prime 2}-u^{3} v^{3} h^{\prime 2}+5 u^{2} v^{2} h h^{\prime 2}-\frac{11}{2} u v^{2} h^{2} h^{\prime 2}+u^{2} v h^{2} h^{\prime 2} \\
+ & \left.u^{2} v^{2} h^{2} h^{\prime}+u^{2} v^{3} h h^{\prime 2}+u^{2} v^{3} h^{2} h^{\prime}\right) h^{\prime \prime}+\left(2 u^{4}-2 h u^{3}\right. \\
+ & 4 v h^{3}-2 u^{3} v+8 u^{2} v h-10 u v h^{2}-8 u v^{3} h-2 u^{4} h^{\prime} \\
+ & 4 u^{3} h h^{\prime}+6 v^{3} h^{2}+2 v^{5} h h^{\prime}+2 u^{2} v^{3}-2 u^{3} v^{2}-2 u v^{5} h^{\prime} \\
+ & 2 u^{2} v^{2} h-4 u v^{3} h h^{\prime}-2 u^{2} h^{2} h^{\prime}+2 v^{3} h^{2} h^{\prime}+2 u^{2} v^{3} h^{\prime} \\
&
\end{aligned}
$$


$u, v \in \mathbb{R} \backslash\{0\}$

$$
\begin{aligned}
& +2 u^{2} v^{2} h-4 u v^{3} h h^{\prime}-2 u^{2} h^{2} h^{\prime}+2 v^{3} h^{2} h^{\prime}+2 u^{2} v^{3} h^{\prime} \\
& +2 u^{3} v^{2} h^{\prime}-2 u^{2} v^{2} h h^{\prime}+4 u^{3} h-4 v^{4} h-2 u^{3} v+2 u v^{4}-8 u v^{2} h \\
& +4 u^{2} v h+12 u v^{3} h-8 u^{2} h^{2}+8 v^{2} h^{2}+4 v^{4} h h^{\prime}+2 u^{2} v^{2} \\
& -v^{6} h^{\prime}-6 u^{2} v^{3}+4 u^{3} v^{2}-2 u v^{4} h^{\prime}+4 u v^{5} h^{\prime}+4 u v^{2} h h^{\prime}+4 u^{2} v h h^{\prime} \\
& -8 u^{2} v^{2} h-8 u v h^{2} h^{\prime}-4 u v^{3} h h^{\prime}-2 u^{4} h^{\prime}-u^{4} h^{\prime 3}-v^{6} h^{\prime 3} \\
& +4 u^{3} h h^{\prime}+4 v^{4} h h^{\prime}+2 u^{3} v h^{\prime}-2 u v^{4} h^{\prime}-4 u^{2} v h h^{\prime}-4 u v^{3} h h^{\prime} \\
& +2 v^{6} h^{\prime 2}+4 u^{3} h h^{\prime 2}+2 u v^{4} h^{\prime 2}-4 u v^{5} h^{\prime 2}+4 u^{2} v h h^{\prime 2}-4 u v^{3} h h^{\prime 2} \\
& -4 u^{2} h^{2} h^{\prime}-4 v^{4} h h^{\prime 2}-2 u^{3} v h^{\prime 4}-u^{2} v^{2} h^{\prime}+4 u^{2} v^{3} h^{\prime}-4 u^{2} v^{4} h^{\prime} \\
& \left.-8 u^{2} v^{2} h h^{\prime}+2 u^{2} v^{3} h^{\prime}+2 u^{2} v^{3} h^{\prime 3}-2 u^{2} v^{3} h^{\prime 2}+4 u^{3} v^{2} h^{\prime 2}\right) h^{\prime} \\
& \xi(u, v) \neq 0, \\
& D=-\left(-u^{2} h^{\prime}+2 u h-u^{2}\right)^{2}
\end{aligned}
$$

Proof. We consider a rotational surface (10). Coefficients of the first and the second fundamental forms are

$$
\begin{aligned}
& E=0, \quad F=-u^{2} h^{\prime}+2 u h-u^{2}, \quad G=(-u+h)^{2}, \\
e= & \frac{1}{\sqrt{|D|}}\left[\left(-2 u^{2} v+3 \cdot 2^{-1} u v^{2}\right) h^{\prime \prime}+2 v^{2} h h^{\prime} h^{\prime \prime}\right. \\
& +\left(u^{2} v-3 \cdot 2^{-1} u v^{2}+2^{-1} v^{4}\right) h^{\prime} h^{\prime \prime}+\left(u^{2} v-2^{-1} u v^{2}+1\right) h h^{\prime \prime} \\
& \left.+2\left(-u^{2}+v^{3}\right) h^{\prime}+4 v h+2 u(u-v)\right], \\
f= & \frac{1}{\sqrt{|D|}}\left[\left(-u^{2}+v^{3}\right) h^{\prime 2}+2(u+v) h h^{\prime}+\left(-u v+2 u v^{2}-v^{3}\right) h^{\prime}\right. \\
& +2(u-v) h+u(v-u)], \\
g= & \frac{1}{\sqrt{|D|}}\left[\left(u^{2}-u v^{2}\right) h^{\prime}+h^{2} h^{\prime}+\left(-2 u+v^{2}\right) h h^{\prime}+h^{2}-u h\right] .
\end{aligned}
$$

Since $D=-\left(-u^{2} h^{\prime}+2 u h-u^{2}\right)^{2}<0$ then $R(u, v)$ is a timelike surface. The mean curvature and the Gaussian curvature of a TRS with LPC of $(L, L)$-type are given as follows, respectively,

$$
\begin{aligned}
& H=\frac{\varsigma(u, v)}{2 D^{3 / 2}} \\
& K=\frac{\xi(u, v)}{D^{2}}
\end{aligned}
$$

where $D=-\left(-u^{2} h^{\prime}+2 u h-u^{2}\right)^{2}, u, v \in \mathbb{R} \backslash\{0\}$. Hence, we can easily see the relation between $H$ and $K$. 


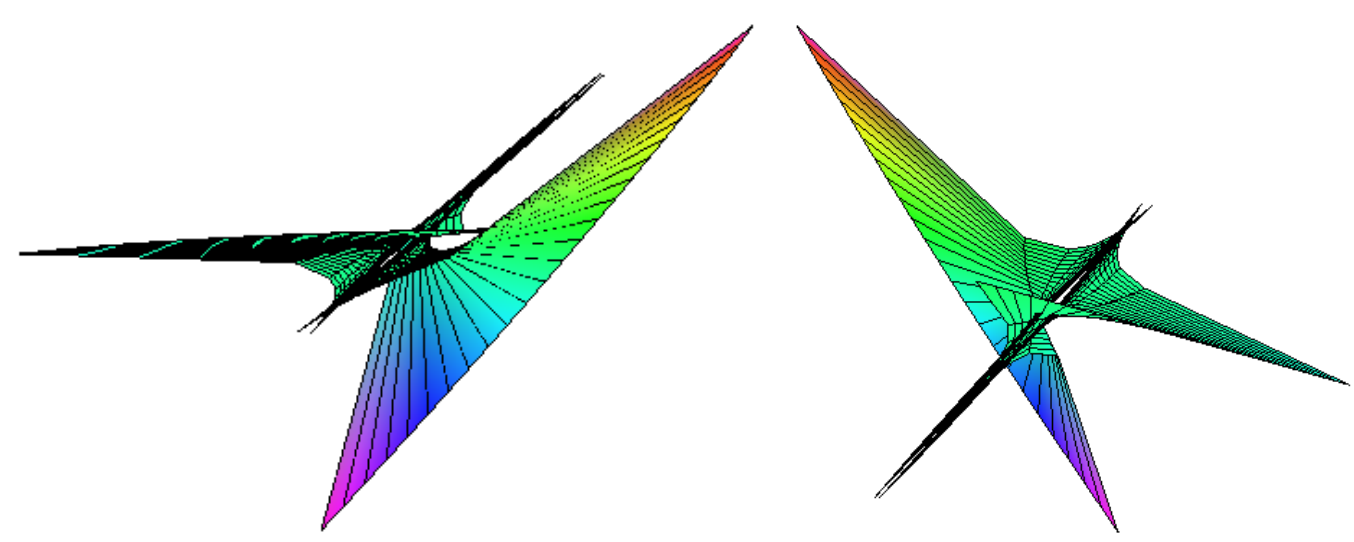

Figure 11. a-b $\Delta N$ of a TRS with LPC of (L,L)-type.

Theorem 4.8. The Gauss map and its Laplacian (see Fig. 11) of a TRS with $L P C$ of $(L, L)$-type are related as

$$
\Delta \mathbf{N}=\Gamma+\mathbf{N}
$$

in Minkowski 3-space, where $\mathbf{N}=\mathbf{N}(u, v)$ in (12),

$$
\begin{aligned}
& \Gamma=\Gamma(u, v)=2 D^{-2}\left\{-2\left[\left(G_{u}-F_{v}\right) \mathbf{N}_{u}-F_{u} \mathbf{N}_{v}+G \mathbf{N}_{u u}-2 F \mathbf{N}_{u v}\right] D\right. \\
& \left.+\left(G \mathbf{N}_{u}-F \mathbf{N}_{v}\right) D_{u}-F \mathbf{N}_{u} D_{v}-2 D^{3 / 2} \mathbf{N}\right\} \text {, } \\
& D=-\left(-u^{2} h^{\prime}+2 u h-u^{2}\right)^{2}, u, v \in R \backslash\{0\}
\end{aligned}
$$

Proof. We use the Gauss map of a TRS with LPC of $(L, L)$-type in (12). Therefore, from (1) and using the same methods in theorem 4.2 and 4.5.we get easily $\Delta \mathrm{N}$. 

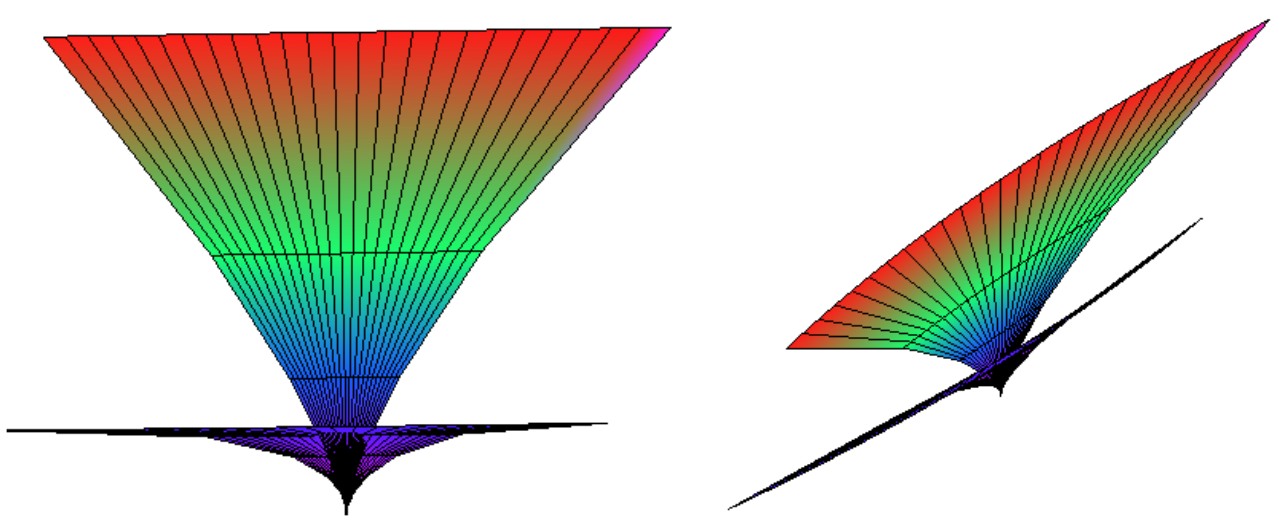

Figure 12. a-b $\Delta R$ of a TRS with LPC of (L,L)-type.

Theorem 4.9. A TRS with LPC of $(L, L)$-type and its Laplacian (see Fig. 12) are related as

$$
\Delta R=\Lambda+R
$$

in Minkowski 3-space, where $R=R(u, v)$ in (10),

$$
\begin{aligned}
& \Lambda(u, v)=\left(\Lambda_{1}, \Lambda_{1}, \Lambda_{3}\right), \\
& \Lambda_{1}= 2 u\left(u^{4}-u^{3}-2 u^{3} h+u^{2} h^{2}+3 u^{2} v h-3 u h^{2}+v h^{3}\right) h^{\prime \prime} \\
&+u^{2}\left(u^{6}-u^{5} v+u^{4} v h+2 u^{2}+2 u-2 v h\right) h^{3}+u\left(3 u^{7}\right. \\
&-6 h u^{6}-3 u^{6} v+9 u^{5} v h-6 u^{4} v h^{2}+6 u^{3}-2 u^{2} v-12 u^{2} h \\
&\left.-2 u v h+4 v h^{2}\right) h^{\prime 2}+\left(3 u^{8}-12 u^{7} h-3 u^{7} v+15 u^{6} v h\right. \\
&+12 u^{6} h^{2}-24 h^{2} u^{5} v+12 u^{4} v h^{3}-4 u^{4}-4 u^{3} h+14 u^{2} h^{2} \\
&\left.+4 u^{2} v h-2 u v h^{2}-2 v h^{3}\right) h^{\prime}-8 u^{3} v h^{4}+\left(-8 u^{5}+20 u^{4} v\right. \\
&\left.+2 v h^{3}\right) h^{3}+2 u\left(6 u^{5}-9 u^{4} v-7 u-v\right) h^{2}+u^{3}\left(7 u^{3} v-6 u^{2}\right. \\
&+16) h+u^{4}\left(u^{4}-u^{3} v-4\right), \\
& \Lambda_{2}= u\left(2 u^{4} v+u^{3}-u^{3} v^{2}-4 u^{3} v h+3 u^{2} v^{2} h-2 u^{2} h+2 u^{2} v h^{2}\right. \\
&\left.-3 u v^{2} h^{2}+u h^{2}+v^{2} h^{3}\right) h^{\prime \prime}+u^{7}+u^{2} v\left(-v h+u v^{2}+2 u^{2} v\right. \\
&\left.+u^{6}+\frac{1}{2} u^{4} v h-\frac{1}{2} u^{5} v\right) h^{3}+u\left(+3 u^{7} v+3 u^{6}-6 u^{6} v h\right. \\
&-\frac{3}{2} u^{6} v^{2}-6 u^{5} h+\frac{9}{2} u^{5} v^{2} h-3 u^{4} v^{2} h^{2}+4 u^{4}+6 u^{3} v \\
&\left.-u^{2} v^{2}+2 u^{2}-12 u^{2} v h-u v^{2} h-2 u h+2 v^{2} h^{2}\right) h^{\prime 2}
\end{aligned}
$$




$$
\begin{aligned}
& +\left(3 u^{8} v-12 u^{7} v h-\frac{3}{2} u^{7} v^{2}+3 u^{7}-12 u^{6} h+12 u^{6} v h^{2}\right. \\
& +\frac{15}{2} u^{6} v^{2} h+12 u^{5} h^{2}-12 u^{5} v^{2} h^{2}+8 u^{5}-16 u^{4} h \\
& -4 u^{4} v+6 u^{4} v^{2} h^{3}-4 u^{3} v h+14 u^{2} v h^{2}-4 u^{2} h+2 u^{2} v^{2} h \\
& \left.-u v^{2} h^{2}+4 u h^{2}-v^{2} h^{3}\right) h^{\prime}-4 u^{3} v^{2} h^{4}+\left[2 u ^ { 4 } \left(-4 u v+5 v^{2}\right.\right. \\
& \left.-4)+v^{2}-2\right] h^{3}+u\left(12 u^{5} v+12 u^{4}-9 u^{4} v^{2}+16 u^{2}\right. \\
& \left.-14 u v-v^{2}\right) h^{2}+u^{3}\left(-6 u^{4} v-6 u^{3}+\frac{7}{2} u^{3} v^{2}-16 u\right. \\
& +16 v) h+u^{4}\left(u^{4} v+u^{3}-\frac{1}{2} u^{3} v^{2}+4 u-4 v\right),
\end{aligned}
$$

$$
\begin{aligned}
\Lambda_{3}= & u\left(-u^{3}+4 u^{2} h-4 u^{3} v h-3 u v^{2} h^{2}+2 u^{2} v h^{2}-u^{3} v^{2}+2 u^{4} v\right. \\
& \left.-5 u h^{2}+2 h^{3}+3 u^{2} v^{2} h+v^{2} h^{3}\right) h^{\prime \prime}+u^{2}\left(u^{6} v-\frac{1}{2} u^{5} v^{2}\right. \\
& \left.+\frac{1}{2} u^{4} v^{2} h+u^{4} h+2 u^{2} v+2 u+u v^{2}-v^{2} h-2 h\right) h^{3} \\
& +u\left(3 u^{7} v-6 u^{6} v h-\frac{3}{2} u^{6} v^{2}+3 u^{5} h+\frac{9}{2} u^{5} v^{2} h-3 u^{4} v^{2} h^{2}\right. \\
& -6 u^{4} h^{2}+4 u^{4}+6 u^{3} v-u^{2} v^{2}-12 u^{2} v h-u v^{2} h-4 u h \\
& \left.+2 v^{2} h^{2}+4 h^{2}\right) h^{2}+\left(3 u^{8} v-12 u^{7} v h-\frac{3}{2} u^{7} v^{2}\right. \\
& +\frac{15}{2} u^{6} v^{2} h+3 u^{6} h+12 u^{6} v h^{2}-12 u^{5} h^{2}-12 u^{5} v^{2} h^{2} \\
& +8 u^{5}+6 u^{4} v^{2} h^{3}+12 u^{4} h^{3}-16 u^{4} h-4 u^{4} v-4 u^{3} v h \\
& \left.+14 u^{2} v h^{2}+2 u^{2} v^{2} h-u v^{2} h^{2}+2 u h^{2}-2 h^{3}-v^{2} h^{3}\right) h^{\prime} \\
& +\left(-4 u^{3} v^{2}-8 u^{3}\right) h^{4}+\left(-8 u^{5} v+12 u^{4}+10 u^{4} v^{2}+v^{2}\right) h^{3} \\
& +u\left(12 u^{5} v-9 u^{4} v^{2}-6 u^{4}+16 u^{2}-14 u v-v^{2}\right) h^{2} \\
& +u^{3}\left(-6 u^{4} v+u^{3}+\frac{7}{2} u^{3} v^{2}-16 u+16 v\right) h+u^{4}\left(u^{4} v\right. \\
& \left.-\frac{1}{2} u^{3} v^{2}+4 u-4 v\right),
\end{aligned}
$$

$u, v \in \mathbb{R} \backslash\{0\}$ and $D=-\left(-u^{2} h^{\prime}+2 u h-u^{2}\right)^{2}$.

Proof. Using Eq. (1), (2) and (10), then we can easily see the Laplacian of a TRS with LPC of $(L, L)$-type. 
ÖZET: Bu çalışmada, $(S, L),(T, L)$ ve $(L, L)$-türünde olan lightlike üreteç eğrili timelike dönel yüzeylerin bazı geometrik özelikleri Minkowski 3-uzayında gösterildi.

\section{REFERENCES}

[1] Beneki, Chr. C., Kaimakamis, G. and Papantoniou, B. J. Helicoidal surfaces in three dimensional Minkowski space, J. Math. Anlys. Appl. 275, (2002) 586-614.

[2] Bour, E. Memoire sur le deformation de surfaces, Journal de l'Êcole Polytechnique, XXXIX Cahier 1-148 (1862).

[3] Güler, E. Bour's theorem and lightlike profile curve, Yokohama Math. J. 54-1, (2007) 55-77.

[4] Hacısalihoğlu, H. H. Yüksek Diferensiyel Geometriye Giriş, Firat Üniversitesi Fen Fakültesi Yayınları, Mat-No.2 (2006).

[5] Hacısalihoğlu, H. H. and Ekmekçi, N. Tensör Geometri, Ankara Üniversitesi Fen Fakültesi Yayınları, (2003).

[6] Ikawa, T. Bour's theorem and Gauss map, Yokohama Math. J. 48, (2000) 173-180.

[7] Ikawa, T. Bour's theorem in Minkowski geometry, Tokyo J. Math. 24, (2001) 377-394.

[8] Niang, A. On rotation surfaces in the Minkowski 3 dimensional space pointwise 1-type Gauss map, J. Korean Math. Soc., 41-6 (2004) 1007-1021.

[9] O 'Neill, B. Semi Riemannian Geometry, Academic Press, New York (1983).

Current address: Erhan GÜLER, Anafartalar Commercial, Vocational High School, Ankara, TURKEY

H.Hilmi HACISALİHOĞLU, Bilecik University, Faculty of Sciences, Department of Mathematics, Bilecik, TURKEY,

E-mail address: ergler@gmail.com, hacisali@science.ankara.edu.tr

$U R L:$ http://communications.science.ankara.edu.tr 